\title{
A case of a patient positive for anti-cardiolipin antibodies with recurrent fetal wastage and cerebral infarction who was successfully treated with Sairei-to, low dose aspirin, and heparin
}

\author{
Taro Nonaka', Makoto Chihara ${ }^{1}$, Izumi Ooki ${ }^{1}$, Masaki Tamura ${ }^{2}$, Takumi Kurabayashi' ${ }^{2}$, \\ Koichi Takakuwa ${ }^{*}$ \\ ${ }^{1}$ Department of Obstetrics and Gynecology, Niigata University General Hospital of Medical and Dental Sciences, Niigata, Japan \\ ${ }^{2}$ Department of Obstetrics and Gynecology, Niigata City General Hospital, Niigata, Japan \\ Email: "obgy@med.niigata-u.ac.jp, tarononaka1029@yahoo.co.jp, chihara-m@med.niigata-u.ac.jp, ooki@honda-lc.com, \\ masakita@hosp.niigata.niigata.jp, takumi@hosp.niigata.niigata.jp
}

Received 29 August 2012; revised 30 September 2012; accepted 10 October 2012

\begin{abstract}
We report a successful case of a pregnant female positive for anti-cardiolipin antibodies who experienced two abnormal pregnancies with postpartum cerebral hemispheric infarctions. A 38-year-old female diagnosed as being positive for anti-cardiolipin antibodies was referred to our hospital due to her strong desire to have a baby. The administration of Japanese herbal medicine, Sairei-to, as immunosuppressive therapy, and low dose aspirin, as anti-coagulation therapy, were initiated prior to the patient's pregnancy. Five months after beginning the treatment, the patient conceived spontaneously. At 34 weeks of gestation, emergency cesarean section was performed due to increasing genital bleeding resulting from coincidental placenta previa and the patient delivered an appropriate-for-date female infant (1970 g). Treatment with Japanese herbal medicine (Saireito) and low-dose aspirin is considered to be an effective treatment option for patients positive for antiphospholipid antibodies with past histories of abnormal pregnancies.
\end{abstract}

Keywords: Anti-Cardiolipin Antibody; Postpartum Cerebral Hemispheric Infarction; Abnormal Pregnancy; Sairei-to; Low Dose Aspirin

\section{INTRODUCTION}

In the past two decades, the autoimmune mechanism underlying reproductive failures and thrombotic events during pregnancy has been recognized and accepted to be anti-phospholipid syndrome [1-6]. Although some

*Corresponding author. cases of anti-phospholipid syndrome are difficult to manage, we have previously reported regarding the efficacy of treatment with Japanese herbal medicine (Saireito) and low-dose aspirin, with or without the addition of prednisolone, in patients positive for anti-phospholipid antibodies [7-9]. This is a case report of a successfully treated pregnant female positive for anti-cardiolipin antibodies who experienced two abnormal pregnancies with postpartum cerebral hemispheric infarction.

\section{CASE}

A parous female was referred to our hospital because she had a strong desire to have a baby. The patient had previously experienced two abnormal pregnancies. During the first pregnancy, severe type pre-eclampsia and intrauterine fetal growth restriction (IUGR) were observed at 23 weeks of gestation, and a diagnosis of idiopathic thrombocytopenic purpura (ITP) was made due to a low platelet count and the appearance of stomatorrhagia. The patient received a transfusion of platelet concentrates and gamma-globulin therapy to treat the ITP. At 23 weeks and six days of gestation, the patient underwent emergency cesarean section due to an aggravation of the pre-eclampsia, and delivered an infant weighing $356 \mathrm{~g}$. The infant had Apgar scores of 1 point at one minute and 3 points at five minutes and died three days after the delivery due to respiratory insufficiency. Four months after the delivery, the patient underwent splenectomy to treat the ITP.

Seven months after the first delivery, the patient spontaneously conceived a second time. During the second pregnancy, IUGR was observed at 19 weeks of gestation and pre-eclampsia developed at 21 weeks of gestation. Fetal growth arrest occurred for one month and was fol- 
lowed by an intrauterine fetal death (IUFD). At 25 weeks and three days of gestation, labor was induced and the patient vaginally delivered an infant weighing $195 \mathrm{~g}$. Ten days after the delivery, a left side cerebral hemispheric infarction suddenly developed, and the patient was diagnosed with left side cerebral thrombosis by magnetic resonance imaging (MRI) (Figure 1) and received conservative anti-coagulation therapy. The patient recovered completely from the cerebral infarction with treatment with anti-coagulation therapy.

Although the attending doctors were reluctant to give the patient permission to conceive a third time because of the serious outcomes of her prior pregnancies, the patient continued to have a strong desire to have a baby and was referred to our hospital. First, the causes of the patient's recurrent abnormal pregnancies were examined. No uterine (mullerian) anomalies were detected and the karyotypes of both the patient and her husband were normal. Tests for autoimmunity revealed the patient to be positive for anti-cardiolipin antibodies, antinuclear antibodies, and the antibodies of Sjogren syndrome A. The data obtained were as follows: anti-cardiolipin antibody, 42 GPL (cutoff value, 33); anti-nuclear antibody, 30 $\mathrm{IU} / \mathrm{ml}$ (cutoff value, 20); anti-SSA antibody, 116 index (cutoff value, 30 ). We concluded that the patient's previous adverse pregnancies and cerebral hemispheric infarctions were caused by these autoimmune abnormalities, in particular by the presence of anti-cardiolipin antibodies, and that medical intervention would be necessary to improve the prognosis of the patient's pregnancy. Informed consent was obtained from the patient before starting the treatment.

The administration of Japanese herbal medicine, Sairei-to (9 g daily), as immunosuppressive therapy, and low dose aspirin (81 mg daily), as anti-coagulation therapy, were initiated prior to the patient's pregnancy (Figure 2). Five months after beginning the treatment, the patient conceived spontaneously. The levels of anti-cardiolipin antibodies in the patient's blood gradually decreased with the administration of Sairei-to. At nine weeks of gestation, 10,000 units of unfractionated heap-

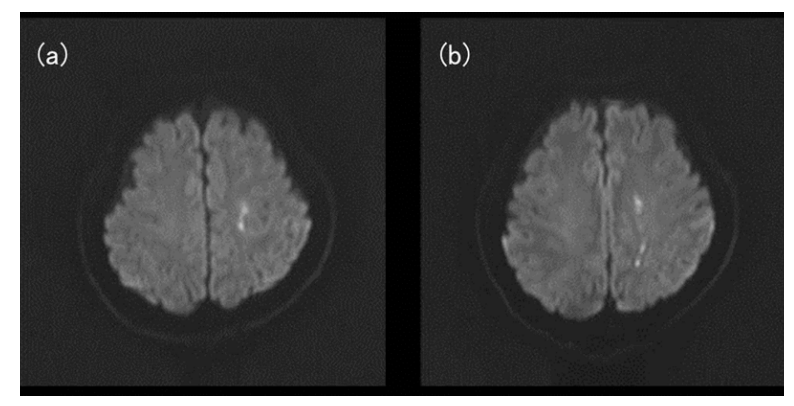

Figure 1. MRI (diffusion weighted image) of the brain. High-intensity areas in left parietal lobe (a) and occipital lobe (b) suggested left side cerebral thrombosis.

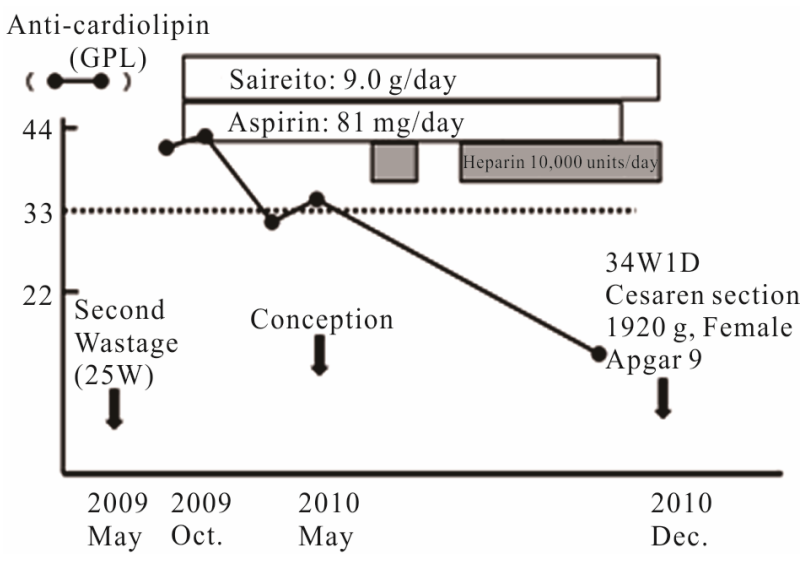

Figure 2. The clinical course of the case. The closed circles indicate the alterations in the level of anti-cardiolipin antibodies, which decreased with the administration of Sairei-to.

rin were administered daily, in addition to Sairei-to, in order to prevent thrombosis. At 13 weeks of gestation, the administration of heparin, however, was stopped, as thrombocytopenia (platelet count, $87,000 / \mathrm{mm}^{3}$ ) was observed, which indicated the development of heparininduced thrombocytopenia (HIT). The patient's platelet count just prior to the start of heparin administration was measured to be $168,000 / \mathrm{mm}^{3}$. However, a diagnosis of HIT was ruled out because the patient tested negative for anti-HIT-antibodies, and heparin administration was restarted at 17 weeks of gestation. Although the patient's platelet counts measured between 80,000 to $100,000 / \mathrm{mm}^{3}$ thereafter, no hemorrhagic diathesis was observed.

The status of the fetus was ascertained to be positive and fetal growth was appropriate during the course of the pregnancy. Although the general condition of the patient was stable thereafter, placenta previa was diagnosed using transvaginal ultrasonography at 30 weeks of gestation. The patient underwent cesarean section at 34 weeks of gestation due to increased genital bleeding, and an appropriate-for-date female infant (1970 g) was born with an Apgar score of 9 points one minute after the delivery. Although the patient's platelet count at the time of cesarean section measured $60,000 / \mathrm{mm}^{3}$ and a transfusion of platelet concentrates was performed, the amount of blood loss during surgery measured $930 \mathrm{ml}$ and no serious complications were observed. Both the puerperal course of the patient and the postnatal course of the infant in the neonatal intensive care unit were uneventful. The administration of Sairei-to continued until delivery, while the administration of low-dose aspirin was terminated at 33 weeks of gestation in order to prepare for emergency cesarean section. The injection of heparin was stopped three hours before cesarean section, and was re-started after the surgery. The administration of warfarin potassium was started on the 7th day after cesarean section as a substitute for heparin. At the obstetric and 
pediatric follow-up conducted six months after the delivery, both mother and child were doing well. Nine months after delivery, the administration of low-dose aspirin was initiated as a substitute for warfarin potassium, as no thrombophilic signs or symptoms were noted.

\section{DISCUSSION}

It is widely accepted in the fields of reproductive immunology and perinatal medicine that anti-phospholipid antibodies are generative factors for a range of reproductive failures, such as recurrent abortions, IUGR, IUFD, and pre-eclampsia [1-6]. In this case, the presence of anti-cardiolipin antibodies, as well as that of other autoantibodies, such as anti-nuclear antibodies and anti-SSA antibodies, was observed, which strongly indicated that the complications that occurred during the patient's past two abnormal pregnancies, including pre-eclampsia, IUGR, and postpartum cerebral hemispheric infarctions, had occurred due to the presence of anti-phospholipid antibodies.

As a treatment for patients positive for anti-phospholipid antibodies with a diversity of adverse pregnancies, the efficacy of combining adrenal corticosteroid hormones and low-dose aspirin therapy has been studied [10]. Although the combination of heparin and low-dose aspirin therapy has been favored in recent reports [11,12], pregnancy outcomes obtained with this treatment modality are not necessarily satisfactory $[13,14]$.

The main mechanisms involved in the generation of reproductive failures are considered to be the occurrence of direct damage to the chorionic villi caused by antiphospholipid antibodies during the period of placentation [15-18], and the formation of thrombi intervillous spaces [19]. Considering these mechanisms, the administration of immune suppressive therapy in combination with anti-coagulation therapy is considered to be a viable treatment option. Japanese herbal medicine, Sairei-to, is reported to have pharmacologic effects similar to those of adrenal corticosteroid hormones, including similar immunosuppressive and anti-inflammatory effects [2023]. Additionally, we have previously reported that treatment with Sairei-to and low dose aspirin, adding prednisolone as needed, is efficacious in patients with prior adverse complications during pregnancy, such as severe pre-eclampsia, placental abruption, HELLP syndrome, or fetal wastages positive for anti-phospholipid antibodies [9].

In this context, treatment with Sairei-to and low dose aspirin was applied in this case before the patient's pregnancy. In addition to this therapy, heparin was administered starting at nine weeks of gestation in order to prevent thrombosis. Although the pregnancy resulted in preterm labor, as a co-incidental complication of placenta previa, at 34 weeks of gestation, an appropri- ate-for-date infant was born. The clinical course of this case clearly indicates that the treatment was effective for this patient. In particular, treatment with Sairei-to and low-dose aspirin was demonstrated to be very effective. The efficacy of heparin in this case, however, was unclear, as this treatment was temporarily stopped from 13 to 17 weeks of gestation, a period which is thought to be crucial for the completion of placentation, in consideration of the appearance of heparin-induced thrombocytopenia (HIT).

Although this is a single case report, the clinical course of this case strongly indicates that immunosuppressive therapy and anti-coagulation therapy are important for the management of patients positive for antiphospholipid antibodies with prior adverse complications during pregnancy, such as severe pre-eclampsia, IUGR, and IUFD. Future studies comparing the efficacy of immunosuppressive and anti-coagulation therapy (Sairei-to and low-dose aspirin) with anti-coagulation specific therapy (heparin and low-dose aspirin) are warranted in order to identify the most appropriate treatment for such patients.

\section{REFERENCES}

[1] Hughes, G.R., Harris, E.N. and Gharavi, A.E. (1986) The anticardiolipin syndrome. Journal Rheumatology, 13, 486491.

[2] Triplett, D.A. (1989) Antiphospholipid antibodies and recurrent pregnancy loss. American Journal of Reproductive Immunology, 20, 52-67.

[3] Gleicher, N. (1997) Antiphospholipid antibodies and reproductive failure: What they do and what they do not do; how to, and how not to treat? Human Reproduction, 12, 13-16. doi:10.1093/humrep/12.1.13

[4] Yasuda, M., Takakuwa, K., Tokunaga, A. and Tanaka, K. (1995) Prospective studies of the association between anticardiolipin antibody and outcome of pregnancy. Obstetrics and Gynecology, 86, 555-559. doi:10.1016/0029-7844(95)00247-O

[5] Lockwood, C.J., Romero, R., Feinberg, R.F., Clyne, L.P., Coster, B. and Hobbins, J.C. (1989) The prevalence and biologic significance of lupus anticoagulant and anticardiolipin antibodies in a general obstetric population. American Journal of Obstetrics and Gynecology, 161, 369-373.

[6] Lynch, A., Marlar, R., Murphy, J., Davila, G., Santos, M., Rutledge, J. and Emlen, W. (1994) Antiphospholipid antibodies in predicting adverse pregnancy outcome. A prospective study. Annals of Internal Medicine, 120, 470475.

[7] Takakuwa, K., Yasuda, M., Hataya, I., Sekizuka, N., Tamura, M., Arakawa, M., Higashino, M., Hasegawa, I. and Tanaka, K. (1996) Treatment for patients with recurrent abortion with positive antiphospholipid antibodies using a traditional Chinese herbal medicine. Journal of Perinatal Medicine, 24, 489-494. doi:10.1515/jpme.1996.24.5.489 
[8] Takakuwa, K., Ishii, K., Takaki, Y., Natsume, N., Adachi, H., Kurata, H., Tamura, M., Kurabayashi, T. and Tanaka, K. (2003) Effect of Sairei-to combined with aspirin and prednisolone on four recurrent reproductive failure women who are positive for anti-phospholipid antibodies. American Journal of Chinese Medicine, 31, 659-663. doi:10.1142/S0192415X03001326

[9] Takakuwa, K., Ooki, I., Nonaka, T., Tamura, N., Ishii, K., Kikuchi, A., Tamura, M. and Tanaka, K. (2006) Prophylactic therapy for patients with reproductive failure who were positive for anti-phospholipid antibodies. American Journal of Reproductive Immunology, 56, 237-242. doi:10.1111/j.1600-0897.2006.00421.x

[10] Hasegawa, I., Takakuwa, K., Goto, S., Yamada, K., Sekizuka, N., Kanazawa, K. and Tanaka, K. (1992) Effectiveness of prednisolone/aspirin therapy for recurrent aborters with antiphospholipid antibody. Human Reproduction, 7, 203-207.

[11] Kutteh, W.H. (1996) Antiphospholipid antibody-associated recurrent pregnancy loss: Treatment with heparin and low-dose aspirin is superior to low-dose aspirin alone. Obstetrics and Gynecology, 174, 1584-1589. doi:10.1016/S0002-9378(96)70610-5

[12] Rai, R., Cohen, H., Dave, M. and Regan, L. (1997) Randomized controlled trial of aspirin and aspirin plus heaprin in pregnant women with recurrent miscarriage associated with phospholipid antibodies (or antiphospholipid antibodies). British Medical Journal, 314, 253-257. doi:10.1136/bmj.314.7076.253

[13] Branch, D.W., Silver, R.M., Blackwell, J.L., Reading, J.C. and Scott, J.R. (1992) Outcome of treated pregnancies in women with antiphospholipid syndrome: An update of the Utah experience. Obstetrics and Gynecology, 80, 614620.

[14] Lassere, M. and Empson, M. (2004) Treatment of antiphospholipid syndrome in pregnancy: A systematic review of randomized therapeutic trials. Thrombosis Research, 114, 419-426. doi:10.1016/j.thromres.2004.08.006

[15] Hasegawa, I., Takakuwa, K., Adachi, S. and Kanazawa, K. (1990) Cytotoxic antibody against trophoblast and lymphocytes present in pregnancy with intrauterine fetal growth retardation and its relation to anti-phospholipid antibody. Journal of Reproductive Immunology, 17, 127-

\section{9. doi:10.1016/0165-0378(90)90031-Z}

[16] Katsuragawa, H., Kanzaki, H., Inoue, T., Hirano, T., Mori, T. and Rote, N.S. (1997) Monoclonal antibody against phosphatidylserine inhibits in vitro human trophoblastic hormone production and invasion. Biology of Reproduction, 56, 50-58. doi:10.1095/biolreprod56.1.50

[17] Rote, N.S., Vogt, E., De Vere, G., Obringer, A.R. and Ng, A. (1998) The role of placental trophoblast in the pathophysiology of the antiphospholipid antibody syndrome. American Journal of Reproductive Immunology, 39, 125136. doi:10.1111/j.1600-0897.1998.tb00344.X

[18] Ichikawa, G., Yamamoto, T., Chishima, F., Nakamura, A., Kuno, S., Murase, T. and Suzuki, M. (2011) Effect of anti- $\beta 2$-glycoprotein I antibody on PIGF, VEGF and sVEGFR1 production from cultured choriocarcinoma cell line. Journal of Obstetrics and Gynaecology Research, 37, 1076-1083. doi:10.1111/j.1447-0756.2010.01490.x

[19] Arakawa, M., Takakuwa, K., Honda, K., Tamura, M., Kurabayashi, T. and Tanaka, K. (1999) Suppressive effect of anticardiolipin antibody on the proliferation of human umbilical vein endothelial cells. Fertility and Sterility, 71, 1103-1107. doi:10.1016/S0015-0282(99)00117-X

[20] Kimura, K., Nanba, S., Tojo, A., Matsuoka, H. and Sugimoto, T. (1990) Effect of Sairei-to on the relapse of steroid-dependent nephrotic syndrome. American Journal of Chinese Medicine, 18, 45-50. doi:10.1142/S0192415X90000071

[21] Liu, X.Y. (1995) Therapeutic effect of chan-ling-tang (Sairei-to) on the steroid-dependent nephrotic syndrome in children. American Journal of Chinese Medicine, 23, 255-260. doi:10.1142/S0192415X95000304

[22] Nakano, Y., Suda, T., Tozawa, F., Dobashi, I., Sato, Y., Ohmori, N., Sumitomo, T. and Demura, H. (1993) Saireito (a Chinese herbal drug)-stimulated secretion and synthesis of pituitary ACTH are mediated by hypothalamic corticotropin-releasing factor. Neuroscience Letters, 160, 93-95. doi:10.1016/0304-3940(93)90921-7

[23] Tozawa, F., Dobashi, I., Horiba, N., Sakai, Y., Sakai, K. and Suda, T. (1998) Saireito (a Chinese herbal drug) decreases inhibitory effect of prednisolone and accelerates the recovery of rat hypothalamic-pituitary-adrenal axis. Endocrine Journal, 45, 69-74. doi:10.1507/endocrj.45.69 